\title{
PLANAR PREMIXED-FLAME/END-WALL INTERACTION: THE JUMP CONDITIONS ACROSS THE THIN FLAME*
}

BY

W. B. BUSH (University of California, San Diego)

AND

S. F. FINK (TRW Defense and Space Systems Group)

\begin{abstract}
Within the context of time-dependent interaction of a planar premixed laminar flame with a cold parallel end wall, the jump conditions for the first (spatial) derivatives of the dependent variables across the thin flame are obtained through the solution of the nonlinear diffusive-reactive boundary-value problem that describes the structure of the flame zone. Recently developed numerical techniques are employed to yield solutions of this boundary-value problem.
\end{abstract}

1. Introduction. In the study of the time-dependent interaction of a planar premixed laminar flame with a parallel end wall (Carrier et al. [1]), the model initial/boundaryvalue problem for the unsteady, one-dimensional, low-Mach-number, isobaric, nonisenthalpic interaction of a reacting premixture with a cold, noncatalytic wall is presented. Attention is directed to the determination of the spatial gradients of the dependent variables at the flame front (on both the unburned and burned sides). In [1], a heuristic argument is employed to obtain the jump conditions for these spatial gradients across the thin flame (or reaction zone), although it is stated that these results may be obtained more formally through the application of the techniques of modern asymptotic analysis. The purpose of the present paper is to apply these techniques to review and extend the work in [1] concerning these jump conditions.

The pioneering work on the asymptotic analysis of the jump conditions across a thin flame without interaction with boundaries is that of Zeldovich and Frank-Kamenetskii [2]. Based on the concepts of [2], multiple-scaling techniques of modern asymptotic analysis for the activation-temperature/adiabatic-flame-temperature ratio much greater than unity have now been developed (see Bush and Fendell [3], Carrier et al. [4], Bush [5] for one-dimensional steady analyses; Matkowsky and Sivashinsky [6] for a threedimensional nonsteady analysis). Concurrent with the analysis of the interfacial results for time-dependent one-dimensional flow with interaction with a boundary of [1], an

* Received November 27, 1979; revised version received April 7, 1980. This research was supported by the National Science Foundation under grant ENG 77-18730. The authors gratefully acknowledge the encouragement and kind advice of Dr. G. F. Carrier, Harvard University, and Dr. F. E. Fendell, TRW Systems. The reviewers' constructive criticism and bringing the work of others, particularly [8], to the author's attention are appreciated. 
analysis of these results for steady two-dimensional flow with such interaction was presented by Buckmaster [7].

In Sec. 2, as the point of departure for this analysis, the model initial/boundary-value problem considered in [1] is presented. For the activation temperature ratio much greater than unity, following the work of Bush and Fendell [3] for such a limit, the nonlinear diffusive-reactive boundary-value problem that describes the flow field in the resulting thin flame-front region is formulated, when the generalization is made that the temperature at the flame front may not be equal to the adiabatic flame temperature for all time due to interaction with the wall.

Since there is just a parametric dependence on time in this formulation, the partial differential equation can be effectively reduced to an ordinary differential equation. Such a reduction is given in Sec. 3. Here, also, this boundary-value problem for the reaction zone is compared with that for the premixed flame region of a counterflow diffusion flame (Liñan [8]). In this section, numerical solutions of the reaction-zone problem are obtained, employing techniques developed previously by the authors. Both the dependent variable profiles, for various values of the parameter, and the first derivative of the dependent variable at upstream infinity, as a function of the parameter, are determined. To complement these numerical results, in Sec. 4 analytical solutions for the upstream gradient for limiting values of the parameter are presented.

Since the behavior of the upstream gradient as a function of the parameter can be used most effectively in the specifications of the jump conditions at the flame front when the numerical results can be re-expressed analytically, in Sec. 5 an analytical approximation of the numerical results for this behavior is developed.

In Sec. 6, a brief summary of the results obtained in this paper is presented.

2. Formulation. For the direct, one-step, irreversible chemical reaction between oxidant $O$ and fuel $F$ that generates product $P$, namely:

$$
v_{O} O+v_{F} F \rightarrow v_{P} P
$$

where $v_{i}$ is the stoichiometric coefficient of species $i, i=O, F, P$, the unsteady, onedimensional, low-Mach-number isobaric, nonisenthalpic interaction of this reacting premixture with a cold, noncatalytic wall $(a t \sigma=0)$ is modeled by the following (nondimensional) initial/boundary-value problem (cf. Carrier et al. [1]) in the domain ( $t>0$, $0<\sigma<\infty)$ :

$$
\frac{\partial Y}{\partial t}-\left(\frac{1}{\Psi} \frac{d \Psi}{d t}\right) \sigma \frac{\partial Y}{\partial \sigma}-\frac{1}{\Psi^{2}} \frac{\partial^{2} Y}{\partial \sigma^{2}}=-W, \quad \frac{\partial T}{\partial t}-\left(\frac{1}{\Psi} \frac{d \Psi}{d t}\right) \sigma \frac{\partial T}{\partial \sigma}-\frac{\mathrm{Le}}{\Psi^{2}} \frac{\partial^{2} T}{\partial \sigma^{2}}=W,
$$

with

$$
\begin{array}{lll}
\multicolumn{1}{l}{W=\Lambda Y^{v_{F}}\left(1+\frac{\varphi}{(1-\varphi)} Y\right)^{v o} \exp \mid-\beta \frac{(1-T)}{T} ; ;} \\
Y \rightarrow 0, & T \rightarrow 1 \quad \text { as } \sigma \rightarrow \infty & (t>0) ; \\
\partial Y / \partial \sigma \rightarrow 0, & T \rightarrow 0 \text { as } \sigma \rightarrow 0 & (t>0) ; \\
Y \rightarrow Y_{f} \equiv 0, & T \rightarrow T_{f} \text { as } \sigma \rightarrow 1 & (t>0) ; \\
Y \rightarrow Y_{0}, & T \rightarrow T_{0} \text { as } t \rightarrow 0 & (0<\sigma<\infty) .
\end{array}
$$


Here, (1) $t$ is time, $\sigma$ is the normalized stream function, i.e., $\sigma=\psi / \Psi(t)$, with $\Psi(t)$ the stream function at the flame front; (2) $Y(\sigma, t)$ is the normalized mass fraction of fuel, and $T(\sigma, t)$ is the normalized temperature; (3) Le is the Lewis-Semenov number, of order unity; (4) $W(\sigma, t)$ is the normalized reaction rate, with $\varphi$ the equivalence ratio $(\Phi \equiv(\varphi /(1-\varphi))$ of order unity), $\beta$ the activation temperature ratio, greater than order unity, and $\Lambda$ the "normalized" Damköhler number, greater than order unity. In this model, it is taken that $\Psi(t) \rightarrow \Psi_{0}$, greater than order unity, as $t \rightarrow 0$, and that $T_{f}(t) \rightarrow 1$, as $t \rightarrow 0$. Further, for this model problem, the functions $Y_{0}(\sigma)$ and $T_{0}(\sigma)$ are to be specified consistent with the given boundary conditions.

The spatial gradients of $Y$ and $T$ at the flame front, on the unburned and burned sides, respectively, are denoted by

$$
\begin{array}{lllll}
\frac{\partial Y}{\partial \sigma} \rightarrow\left(\frac{\partial Y}{\partial \sigma}\right)_{f_{-}}, & \frac{\partial T}{\partial \sigma} \rightarrow\left(\frac{\partial T}{\partial \sigma}\right)_{f_{-}} & \text {as } & \sigma \rightarrow 1_{-} & (t \geq 0) ; \\
\frac{\partial Y}{\partial \sigma} \rightarrow\left(\frac{\partial Y}{\partial \sigma}\right)_{f_{+}} \equiv 0, & \frac{\partial T}{\partial \sigma} \rightarrow\left(\frac{\partial T}{\partial \sigma}\right)_{f_{+}} & \text {as } & \sigma \rightarrow 1_{+} & (t \geq 0) .
\end{array}
$$

In order to solve (2.1) in the "thin-flame approximation," i.e. to solve the homogeneous forms of the equations of (2.1a) in the domains $(t>0,0<\sigma<1)$ and $(t>0,1<\sigma<\infty)$ subject to the initial/boundary conditions of $(2.1 \mathrm{~b})-(2.1 \mathrm{~d})$, additional boundary conditions, relating the spatial gradients at the flame front, are required. These flame-front jump conditions are derived below, employing the fact that the activation temperature ratio $\beta$ is greater than order unity for the reaction under consideration.

To study the flame-front region, consider the following transformations of the independent and dependent variables (cf. Bush and Fendell [3] for the analysis of the steady-state counterpart of this problem):

$$
\begin{gathered}
(\sigma, t) \rightarrow(\zeta, t), \quad \text { with } \zeta=\beta(\sigma-1) ; \\
Y(\sigma, t ; \beta)=\beta^{-1} Z(\zeta, t)+O\left(\beta^{-2}\right), \\
T(\sigma, t ; \beta)=T_{f}(t)+\beta^{-1} G(\zeta, t)+O\left(\beta^{-2}\right) .
\end{gathered}
$$

Introduction of the variables of (2.3) into (2.1a) yields, to leading order of approximation, for $(t \geq 0,-\infty<\zeta<\infty)$,

$$
\frac{\partial^{2} Z}{\partial \zeta^{2}}=- \text { Le } \frac{\partial^{2} G}{\partial \zeta^{2}}=J_{0} Z^{v_{F}} \exp \left\{\frac{G}{T_{f}^{2}}\right\}
$$

with

$$
J_{0}=\frac{\Psi^{2}}{2 \Gamma\left(1+v_{F}\right) \mathrm{Le}^{1+v_{F}}} \exp \left\{-\beta \frac{\left(1-T_{f}\right)}{T_{f}}\right\} .
$$

Here, the result of [1] that, to the order of approximation considered, $\Lambda=\left[2 \Gamma\left(1+v_{F}\right) \mathrm{Le}^{1+v_{F}}\right]^{-1} \beta^{1+v_{F}}$, where $\Gamma(k)$ is the (complete) gamma function, has been introduced.

Directly, (2.4) yields

$$
\partial^{2} Z / \partial \zeta^{2}+\operatorname{Le}\left(\partial^{2} G / \partial \zeta^{2}\right)=0
$$


In turn, the first and second integrals of (2.5) are

$$
\frac{\partial Z}{\partial \zeta}+\operatorname{Le} \frac{\partial G}{\partial \zeta}=\Omega, \quad Z+\operatorname{Le} G=\Omega \zeta+\Pi
$$

with $\Omega, \Pi$ functions of integration. From (2.2), (2.3), and (2.6a), it is seen that the spatial gradients at the flame front are related by

$$
\left\lfloor\left(\frac{\partial Y}{\partial \sigma}\right)_{f_{-}}+\operatorname{Le}\left(\frac{\partial T}{\partial \sigma}\right)_{f_{-}}\right\rfloor=\left\lfloor\operatorname{Le}\left(\frac{\partial T}{\partial \sigma}\right)_{f_{+}}\right\rfloor \approx \Omega .
$$

Further, from (2.4) and (2.6b), it is seen that the problem for the flame-front "structure" reduces to the solution of the following equation:

$$
\left.\frac{\partial^{2} Z}{\partial \zeta^{2}}=J_{0} Z^{v_{F}} \exp \mid-\frac{Z}{\operatorname{Le} T_{f}^{2}}\right\} \exp \left\{\frac{\Omega \zeta+\Pi \mid}{\operatorname{Le} T_{f}^{2}} \mid .\right.
$$

To reduce (2.8) to "standard form," the following transformations are introduced:

$$
\begin{gathered}
(\zeta, t) \rightarrow(\xi, t), \quad \text { with } \xi=\frac{\Psi T_{f}^{v_{F}-1}}{\left(\Gamma\left(1+v_{F}\right)\right)^{1 / 2} \operatorname{Le}} \exp \left|-\frac{1}{2}\right| \beta \frac{\left(1-T_{f}\right)}{T_{f}}-\frac{\Pi}{\operatorname{Le} T_{f}^{2}} \mid \zeta ; \\
Z=\operatorname{Le} T_{f}^{2} h .
\end{gathered}
$$

Under these transformations, $(2.8)$ takes the form

$$
2 \frac{\partial^{2} h}{\partial \xi^{2}}=h^{v F} \exp (-h) \exp (M \xi)
$$

with

$$
M=\left(\Gamma\left(1+v_{F}\right)\right)^{1 / 2} \frac{\Omega}{\Psi T_{f}^{1+v_{F}}} \exp \left\{\frac{1}{2}\left|\beta \frac{\left(1-T_{f}\right)}{T_{f}}-\frac{\Pi}{\operatorname{Le} T_{f}^{2}}\right|\right\}
$$

The boundary conditions for this equation, reflecting the requirements that the fuel mass fraction function goes to zero as the burned side of the flame is approached and goes to infinity as the unburned side is approached, are

$$
h \rightarrow 0 \quad \text { as } \xi \rightarrow \infty, \quad h \rightarrow \infty \quad \text { as } \quad \xi \rightarrow-\infty .
$$

The spatial gradients of $h$, as the burned and unburned sides of the flame are approached, are

$$
\frac{\partial h}{\partial \xi} \rightarrow\left(\frac{\partial h}{\partial \xi}\right)_{+}=0 \quad \text { as } \quad \xi \rightarrow \infty, \quad \frac{\partial h}{\partial \xi} \rightarrow\left(\frac{\partial h}{\partial \zeta}\right)_{-}<0 \quad \text { as } \quad \xi \rightarrow-\infty
$$

where $(\partial h / \partial \xi)_{-}$is a function to be determined from the solution of $(2.10)$ and $(2.11)$. In turn, the gradient functions of (2.2) are determined to be

$$
\begin{gathered}
\left(\frac{\partial Y}{\partial \sigma}\right)_{f_{-}} \approx \Omega\left[\frac{1}{M}\left(\frac{\partial h}{\partial \xi}\right)_{-}\right] \equiv-\Omega B, \quad\left(\frac{\partial T}{\partial \sigma}\right)_{f_{-}} \approx \frac{1}{\mathrm{Le}} \Omega(1+B) \\
(\partial Y / \partial \sigma)_{f_{+}}=0, \quad(\partial T / \partial \sigma)_{f_{+}} \approx(1 / \mathrm{Le}) \Omega .
\end{gathered}
$$


3. Solutions. From an examination of (2.10), it is seen that $M=M\left(t ; \beta, v_{F}\right)$, and that $h=h\left(\xi, t ; \beta, v_{F}\right)=h\left(\xi ; M\left(t ; \beta, v_{F}\right), v_{F}\right)$. Thus, for $v_{F}=1$ (a reasonable value for this parameter), the problem of $(2.10)$ and $(2.11)$ can be recast as the following boundaryvalue problem for the determination of $h(\xi ; M)$ :

$$
\begin{aligned}
& 2 \frac{d^{2} h}{d \xi^{2}}=h \exp (-h) \exp (M \xi) \quad(-\infty<\xi<\infty) ; \\
& h \rightarrow 0 \quad \text { as } \quad \xi \rightarrow \infty, \quad h \rightarrow \infty \quad \text { as } \quad \xi \rightarrow-\infty ; \\
& h \rightarrow 1 \quad \text { as } \xi \rightarrow 0 .
\end{aligned}
$$

The boundary condition of (3.1c) is arbitrary, and has been introduced for purposes of mathematical convenience. This boundary condition does not affect the principal result of this analysis, namely: the determination of $(d h / d \xi)$ as $\xi \rightarrow-\infty$. It is seen that (3.1a) is equivalent to (52) of Liñan [8]. However, in [8] (see (53) and (54)), the following boundary conditions (in the notation of the present paper) are applied:

$$
\frac{d h}{d \xi} \rightarrow 0 \quad \text { as } \quad \xi \rightarrow \infty, \quad \frac{d h}{d \xi} \rightarrow-1 \quad \text { as } \quad \xi \rightarrow-\infty .
$$

While the boundary conditions of (3.1b)' may be the appropriate ones for the premixed flame region of a counterflow diffusion flame (Linan [8]), the boundary conditions of (3.1b) are the appropriate ones for the flame-front-region flow problem studied in the present paper.

Under the transformations

$$
\begin{gathered}
\xi \rightarrow \eta, \quad \text { with } \quad \eta=M \xi, \\
h(\xi ; M)=f(\eta ; J), \quad \text { with } \quad J=1 / 2 M^{2},
\end{gathered}
$$

the boundary-value problem of (3.1) can be re-expressed as

$$
\begin{aligned}
& \frac{d^{2} f}{d \eta^{2}}=J f \exp (-f) \exp (\eta) \quad(-\infty<\eta<\infty) ; \\
& f \rightarrow 0 \quad \text { as } \quad \eta \rightarrow \infty, \quad f \rightarrow \infty \quad \text { as } \quad \eta \rightarrow-\infty ; \\
& f \rightarrow 1 \text { as } \eta \rightarrow 0 .
\end{aligned}
$$

Numerical solutions are presented here for the boundary-value problem of (3.3); asymptotic solutions are presented for the boundary-value problem of (3.1).

Preliminary to the obtaining of numerical solutions to the boundary-value problem of (3.3), the behaviors of $f$ as $\eta \rightarrow \pm \infty$ are obtained. The downstream behavior is determined to be

$$
\begin{aligned}
f & \sim b \mathbf{K}_{0}(z)+\cdots \\
& =b\left[\left(\frac{\pi}{2}\right)^{1 / 2} \frac{\exp (-z)}{z^{1 / 2}}(1+\cdots)\right]+\cdots \rightarrow 0 \text { as } z=2 J^{1 / 2} \exp \left(\frac{1}{2} \eta\right) \rightarrow \infty
\end{aligned}
$$


where $\mathbf{K}_{0}(z)$ is the zeroth-order modified Bessel function of the second kind and $b$ is a constant (to be determined). The upstream behavior is determined to be

$$
\begin{aligned}
f \sim(-B \eta & +a)+\frac{J \exp (-a)}{(1+B)^{2}} \\
& \times\left\{(-B \eta+a)+\frac{2 B}{(1+B)}\right\} \exp \{(1+B) \eta\}+\cdots \rightarrow \infty \text { as } \eta \rightarrow-\infty,
\end{aligned}
$$

where $a$ is a constant (to be determined). Further, in (3.5), the notation

$$
\frac{d f}{d \eta} \rightarrow\left(\frac{d f}{d \eta}\right)_{-}=-B=-B(J) \text { as } \eta \rightarrow-\infty
$$

is (re)introduced.

Employing previously-developed numerical techniques, for the values of $J$ listed in Table 1, the boundary-value problem of (3.3) was solved in two steps. The first step involved the solution, by the method of Lentini-Peyrera, of the two-point boundaryvalue problem

$$
\begin{gathered}
d^{2} f / d \eta^{2}=J f \exp (-f) \exp (\eta) \quad(0<\eta<\infty) ; \\
f \rightarrow 0 \quad \text { as } \quad \eta \rightarrow \infty, \quad f \rightarrow 1 \quad \text { as } \quad \eta \rightarrow 0 .
\end{gathered}
$$

From this first step, the first derivative of $f$ at the origin, $-S=-S(J)$, was obtained. Then, the second step involved the solution, by the method of Runge-Kutta, of the initial-value problem

$$
\begin{aligned}
d^{2} f / d \eta^{2} & =J f \exp (-f) \exp (\eta) \quad(-\infty<\eta<0) ; \\
f & \rightarrow 1, \quad d f / d \eta \rightarrow-S \quad \text { as } \quad \eta \rightarrow 0 .
\end{aligned}
$$

From this second step, the first derivative of $f$ at upstream infinity, $-B=-B(J)$, was obtained. The combination of the first and second steps yielded $f$ as a function of $\eta$, for the domain $(-\infty<\eta<\infty)$, for the given values of the parameter $J$. These results are presented in Fig. 1.

As mentioned previously, the principal result to be obtained from these solutions of the boundary-value problem is the determination of $B$ as a function of $J$. This result is presented both in Table 1 and in Fig. 2.

4. Asymptotic solutions. In Appendix $\mathrm{C}$ of [8], the asymptotic forms of the solutions of (3.1a), subject to the boundary conditions of (3.1b)', are presented for (a) $M \rightarrow 0$, (b) $M \rightarrow-\frac{1}{2}$, and (c) $M \rightarrow \infty$. An analysis parallel to that of Appendix C(a) of [8] is presented in Sec. 4.1 below. Since for the present case $M$ is positive, the analysis of Appendix $\mathrm{C}(\mathrm{b})$ is not pertinent here. With appropriate modification (see Sec. 4.2), the analysis of Appendix $\mathrm{C}(\mathrm{c})$ provides further insight for the present analysis concerning the behavior of $B$ as $M \rightarrow \infty$.

4.1. Solutions for $M \rightarrow 0$. To study the limiting case of $M \rightarrow 0$, consider that

$$
h(\xi ; M)=F(\xi)+O(M) .
$$




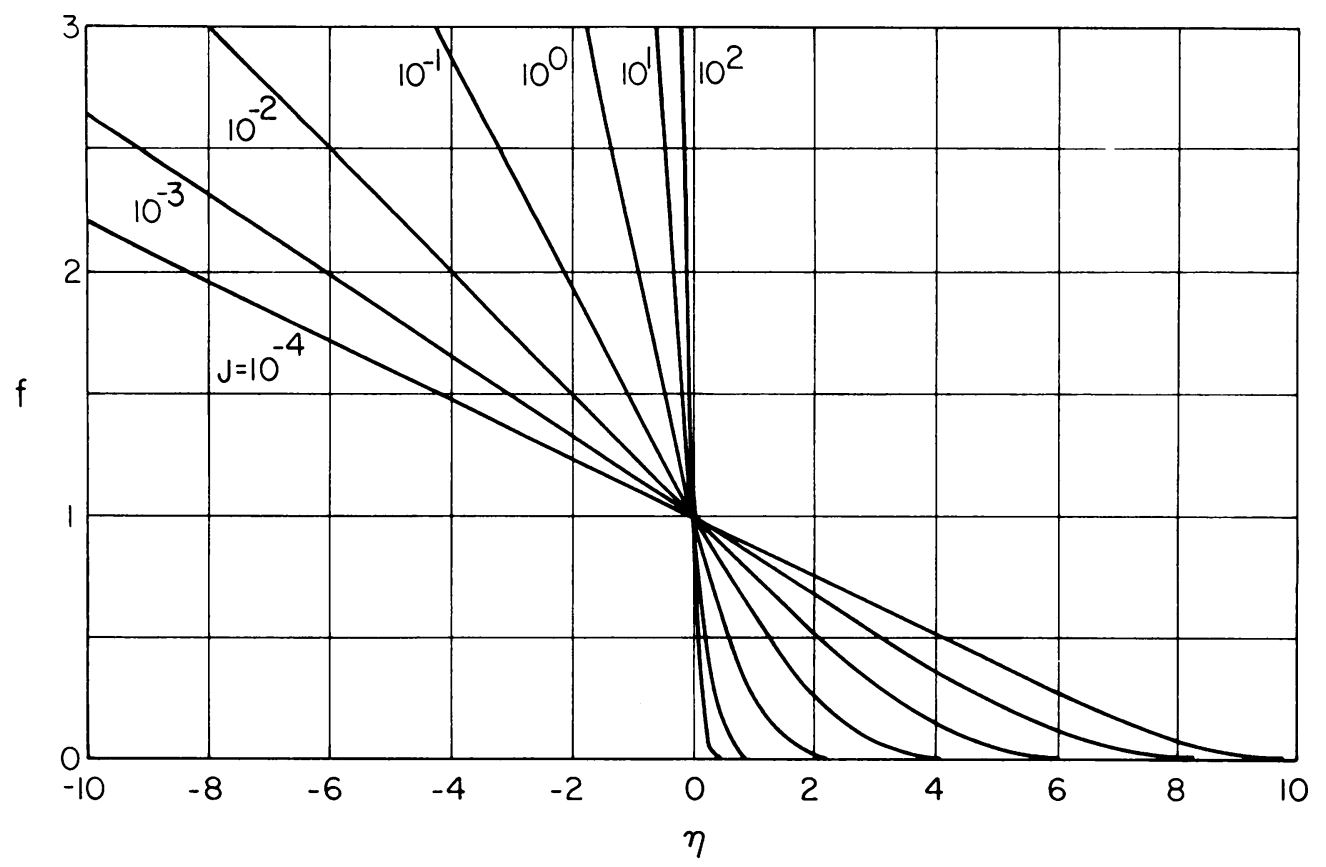

Fig. 1. The numerical solutions of (3.3) for $f$ as a function of $\eta$ for the given values of $J$.

TABLE 1

\begin{tabular}{|c|c|c|c|}
\hline$J$ & $B$ & $B_{0}$ & $B_{0} / B$ \\
\hline $1 \times 10^{-5}$ & $9.461 \times 10^{-2}$ & $9.653 \times 10^{-2}$ & 1.020 \\
\hline $1 \times 10^{-4}$ & $1.202 \times 10^{-1}$ & $1.241 \times 10^{-1}$ & 1.032 \\
\hline $5 \times 10^{-4}$ & $1.477 \times 10^{-1}$ & $1.551 \times 10^{-1}$ & 1.050 \\
\hline $1 \times 10^{-3}$ & $1.637 \times 10^{-1}$ & $1.738 \times 10^{-1}$ & 1.062 \\
\hline $5 \times 10^{-3}$ & $2.170 \times 10^{-1}$ & $2.412 \times 10^{-1}$ & 1.112 \\
\hline $1 \times 10^{-2}$ & $2.510 \times 10^{-1}$ & & \\
\hline $5 \times 10^{-2}$ & $3.807 \times 10^{-1}$ & $B^{0}$ & $B^{0} / B$ \\
\hline $1 \times 10^{-1}$ & $4.572 \times 10^{-1}$ & & \\
\hline $1 \times 10^{0}$ & $1.237 \times 10^{0}$ & $1.414 \times 10^{0}$ & 1.143 \\
\hline $5 \times 10^{0}$ & $2.815 \times 10^{0}$ & $3.162 \times 10^{0}$ & 1.123 \\
\hline $1 \times 10^{1}$ & $4.063 \times 10^{0}$ & $4.471 \times 10^{0}$ & 1.100 \\
\hline $5 \times 10^{1}$ & $9.492 \times 10^{0}$ & $1.000 \times 10^{1}$ & 1.054 \\
\hline $1 \times 10^{2}$ & $1.361 \times 10^{1}$ & $1.414 \times 10^{1}$ & 1.039 \\
\hline
\end{tabular}

Then the boundary-value problem of (3.1) can be re-expressed as

$$
\begin{gathered}
2 \frac{d^{2} F}{d \xi^{2}}=F \exp (-F) \quad(-\infty<\xi<\infty) ; \\
F \rightarrow 0 \quad \text { as } \quad \xi \rightarrow \infty, \quad F \rightarrow \infty \quad \text { as } \quad \xi \rightarrow-\infty .
\end{gathered}
$$

It is seen that (4.2) is the "standard form" of the zeroth-order boundary-value problem for the downstream region of steady-state large-activation-temperature laminar 


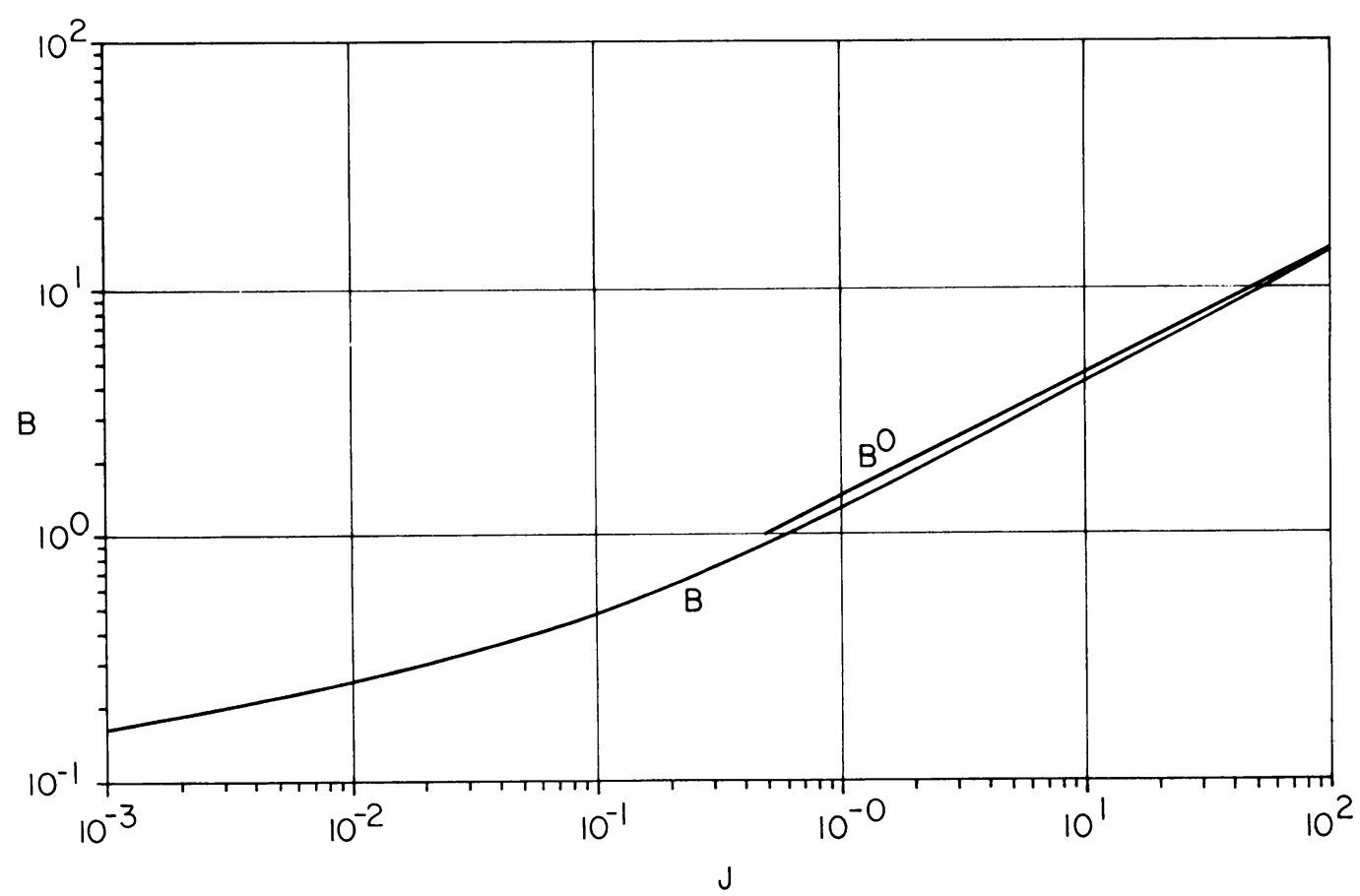

FIG. 2. The determination of $B$ and $B^{0}$ as functions of $J$.

deflagration theory (cf. [3-5]). Multiplication of both sides of (4.2a) by $d F / d \xi$ and integration of the resulting equation, subject to the boundary condition that $d F / d \xi \rightarrow 0$ as $F \rightarrow 0$, yield

$$
d F / d \xi=-[1-(1+F) \exp (-F)]^{1 / 2} .
$$

Integration of (4.3), subject to the boundary condition that $\xi \rightarrow 0$ as $F \rightarrow F_{0}$, yields

$$
\xi=\xi(F)=-\int_{F_{0}}^{F}[1-(1+s) \exp (-s)]^{-1 / 2} d s .
$$

From the above results, it is determined that

$$
d F / d \xi \rightarrow-1 \text { as } \xi \rightarrow-\infty .
$$

Thus, based on the previously-introduced definitions,

$$
\left(\frac{\partial Y}{\partial \sigma}\right)_{f_{-}} \approx \Omega\left[\frac{1}{M}\left(\frac{\partial h}{\partial \xi}\right)_{-}\right] \approx \Omega\left[\frac{1}{M}\left(\frac{\partial F}{\partial \xi}\right)_{-}\right]=-\frac{\Omega}{M},
$$

that is,

$$
B=B(M) \approx 1 / M \quad \text { and } / \text { or } \quad B=B(J) \approx(2 J)^{1 / 2} .
$$

The function $B^{0}(J) \equiv(2 J)^{1 / 2}$ is presented also in Table 1 and Fig. 2 . From these tabular and graphical presentations, it is possible to compare $B(J)$ and $B^{0}(J)$. 
4.2. Solutions for $M \rightarrow \infty$. Since

$$
\begin{aligned}
& h \rightarrow 0 \quad \text { (exponentially) as } \xi \rightarrow \infty, \\
& h \rightarrow-B(M \xi)+a \rightarrow \infty \quad \text { (algebraically) as } \xi \rightarrow-\infty,
\end{aligned}
$$

it is anticipated that, for $B \rightarrow 0$ as $M \rightarrow \infty$,

$$
\exp (-h) \exp (M \xi) \approx \exp (M \xi),
$$

and (3.1a) may be replaced in first approximation by

$$
2 \frac{d^{2} h}{d \xi^{2}}=h \exp (M \xi)
$$

which, in turn, can be written as the Bessel equation

$$
\frac{d^{2} h}{d z^{2}}-\frac{1}{z} \frac{d h}{d z}-h=0,
$$

in terms of the variable

$$
z=\frac{2^{1 / 2}}{M} \exp \left(\frac{1}{2} M \xi\right)
$$

Essentially this transformation is introduced in Sec. 3 to determine the downstream behavior of the dependent variable, where the approximation

$$
\exp (-h) \exp (M \xi) \approx \exp (M \xi)
$$

is appropriate, regardless of the magnitude of $M$. Here, this approximation is taken to hold over the entire range of $h$ for $M \rightarrow \infty$ (and $B(M) \rightarrow 0)$. The solution of (4.8a), satisfying the downstream boundary condition $h \rightarrow 0$ as $z \rightarrow \infty$, is

$$
h=h_{0} \mathbf{K}_{0}(z) \text {, with } h_{0}=\text { const. (to be determined). }
$$

The upstream behavior of the solution of (4.9) is given by

$$
\begin{aligned}
h & \sim h_{0}\left[-\left\{\log \left(\frac{1}{2} z\right)+\gamma_{E}\right\}\left\{1+\left(\frac{1}{2} z\right)^{2}+\cdots\right\}+\left(\frac{1}{2} z\right)^{2}+\cdots\right] \\
& =-B(M \xi)+2 B\left(\log M-\gamma_{E}^{\prime}\right)+\cdots \rightarrow \infty \text { as } z \rightarrow 0, \xi \rightarrow-\infty,
\end{aligned}
$$

where $\gamma_{E} \doteqdot 0.577$ is the Euler constant, $\gamma_{E}^{\prime}=\left(\gamma_{E}-\frac{1}{2} \log 2\right) \doteqdot 0.230$, and where it is taken that $h_{0}=2 B$. For $\xi \rightarrow 0, z \rightarrow 2^{1 / 2} / M \rightarrow 0$, the boundary condition of (3.1c) is satisfied if

$$
h \sim 2 B\left(\log M-\gamma_{E}^{\prime}\right)+\cdots \rightarrow 1,
$$

such that

$$
B=B(M) \approx \frac{1}{2}\left(\log M-\gamma_{E}^{\prime}\right)^{-1} \text { and } / \text { or } \quad B=B(J) \approx \frac{1}{2}\left(\log \left\{(2 J)^{-1 / 2}\right\}-\gamma_{E}^{\prime}\right)^{-1} .
$$

For purposes of comparison, the function $B_{0}(J) \equiv \frac{1}{2}\left(\log \left\{(2 J)^{-1 / 2}\right\}-\gamma_{E}^{\prime}\right)^{-1}$ is presented also in Table 1.

5. Analytical results. To complete the study of the boundary-value problem of (3.3), an analytical approximation, i.e. a curve-fit, of the numerical results for $B(J)$ is con- 


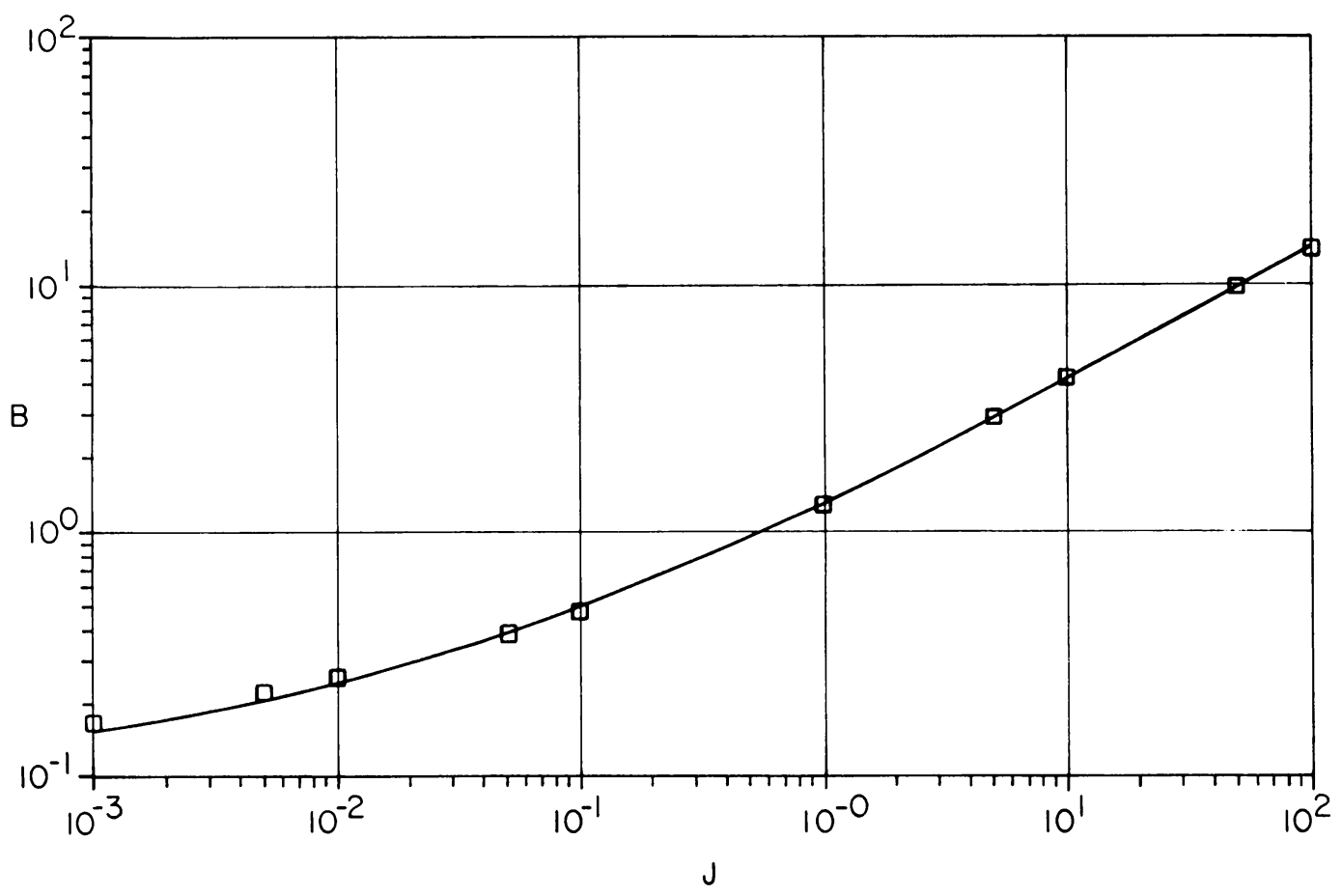

Fig. 3. Analytical approximation of the numerical results for $B$ as a function of $J$. The curve is the approximation of (5.1). The numerical results employed in the determination of this approximation are given.

sidered. To this end, a curve-fitting program based on the Levenburg-Marquardt algorithm was applied to these numerical results.

The following analytical approximation of the numerical data was developed for the indicated range of $J$ :

$$
B \approx\left[\frac{B^{0}}{1+\mu_{1}\left(B^{0}\right)^{-1 / 2}}+\frac{\mu_{2}}{1+\mu_{3} \log \left\{\left(B^{0}\right)^{-1}\right\}}\right] \text { for } 10^{-3} \leq J \leq 10^{2},
$$

with $B^{0}=(2 J)^{1 / 2} ; \mu_{1} \doteqdot 0.506, \mu_{2} \doteqdot 0.263, \mu_{3} \doteqdot 0.293$. The pertinent data and the function of (5.1) are presented in Fig. 3.

6. Summary. In this paper it is determined that the spatial gradients of the fuel mass fraction $Y$ and the temperature $T$ at the flame front on the unburned and burned sides, respectively, are given by

$$
\begin{aligned}
& \left(\frac{\partial Y}{\partial \sigma}\right)_{f_{-}} \approx-\Omega B, \quad\left(\frac{\partial T}{\partial \sigma}\right)_{f_{-}} \approx \frac{1}{\mathrm{Le}} \Omega(1+B), \\
& \left(\frac{\partial Y}{\partial \sigma}\right)_{f_{+}}=0, \quad\left(\frac{\partial T}{\partial \sigma}\right)_{f_{+}} \approx \frac{1}{\mathrm{Le}} \Omega,
\end{aligned}
$$


where, for $v_{F}=1$, the function $B$ is analytically approximated over an extended range of $J$ by

$$
B \approx\left\lfloor\frac{B^{0}}{1+\mu_{1}\left(B^{0}\right)^{-1 / 2}}+\frac{\mu_{2}}{1+\mu_{3} \log \left\{\left(B^{0}\right)^{-1}\right\}}\right\rfloor,
$$

with $\mu_{1}=0.506, \mu_{2}=0.263, \mu_{3}=0.293$, and

$$
B^{0}=(2 J)^{1 / 2}=\frac{1}{M}=\frac{\Psi}{\Omega} T_{f}^{2} \exp \left\{-\frac{1}{2}\left[\beta \frac{\left(1-T_{f}\right)}{T_{f}}-\frac{\Pi}{\operatorname{Le} T_{f}^{2}}\right\} \mid\right.
$$

When it is recalled that, in the original model problem of $(2.1)$, it is taken that $\Psi \rightarrow \Psi_{0} \gg 1$, and that $T \rightarrow 1$ as $t \rightarrow 0$, it is seen that the limit $M \rightarrow 0$ and/or $J \rightarrow \infty$ corresponds to the limit $t \rightarrow 0$. Thus, for small times, the approximation (cf. [1])

$$
\left(\frac{\partial Y}{\partial \sigma}\right)_{f_{-}} \approx-\Psi T_{f}^{2} \exp \left\{-\beta \frac{\left(1-T_{f}\right)}{2 T_{f}}\right\}, \quad \text { for } \quad v_{F}=1,
$$

is justified. The results of (6.1)-(6.2) suggest how the jump conditions can be determined at later times, i.e. $M$ and/or $J \sim O(1)$.

\section{REFERENCES}

[1] G. F. Carrier, F. E. Fendell, W. B. Bush, and P. S. Feldman, Nonisenthalpic interaction of a planar premixed laminar flame with a parallel end wall, SAE Paper No. 790245, presented at SAE Congress and Exposition, Feb. 1979

[2] Y. B. Zeldovich and D. A. Frank-Kamenetskii, On the theory of uniform flame propagation, Dokl. Akad. Nauk SSSR 19, 693-697 (1938)

[3] W. B. Bush and F. E. Fendell, Asymptotic analysis of laminar flame propagation for general Lewis numbers, Comb. Sci. Tech. 1, 421-428 (1970)

[4] G. F. Carrier, F. E. Fendell, and W. B. Bush, Stoichiometry and flame-holder effects on a one-dimensional flame, Comb. Sci. Tech. 18, 33-46 (1978)

[5] W. B. Bush, Asymptotic analysis of laminar flame propagation: review and extension, Int. J. Eng. Sci. 17, 597-613 (1979)

[6] B. J. Matkowsky and G. I. Sivashinsky, An asymptotic derivation of two models in flame theory associated with the constant density approximation, SIAM J. Appl. Math. 37, 686-699 (1979)

[7] J. Buckmaster, The quenching of two-dimensional premixed flames, Acta Astro. 6, 741-769 (1979)

[8] A. Linan, The asymptotic structure of counterflow diffusion flames for large activation energies, Acta Astro. 1, 1007-1039 (1974) 